\section{Pen Marking Technique for Safer Polypectomy with Detachable Snares}

The detachable snare designed by Hachisu [1] is a useful and safe instrument for preventing hemorrhage during and after endoscopic polypectomy for large pedunculated polyps in the colon.

When the detachable snare is being handled, it is important to ensure that it is sufficiently tight when ligating the polyp stalk. If it is too tight, the polyp is transected by the detachable snare alone. If it is not tight enough, the snare can become dislodged, leading to bleeding. Tightening is carried out on the basis of manual assessment by the assistant holding the handle, and depending on the extent of the color change in the polyp caused by congestion. However, it is difficult to select the degree of tightening required.

We devised a method of placing a mark on the silicon stopper from which the loop tightens around the stalk (Figure 1). This mark can be made easily with a pen; we use a red pen, but any conspicuous color can be used. Using this method, the degree of tightness can be confirmed from the marking, in addition to the conventional method of assessing the manual

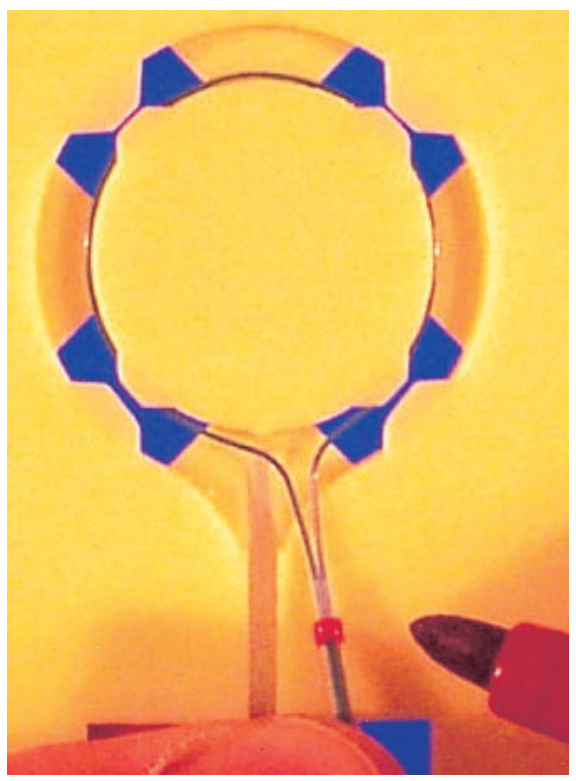

Figure 1 Placing a red mark on the stopper portion of the detachable snare.

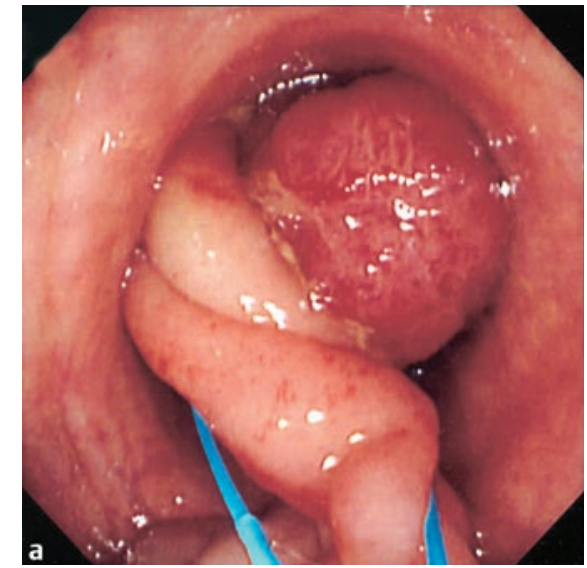

Y. Tomiki, K. Shinmura, T. Maeda, Y. Kojima, K. Terai, S. Kasamaki, Y. Ishibiki, K. Sakamoto, T. Nakajima, T. Kamano

Dept. of Coloproctological Surgery, Juntendo University School of Medicine, Tokyo, Japan.

\section{References}

${ }^{1}$ Hachisu T. A new detachable snare for hemostasis in the removal of large polyps or other elevated lesions. Surg Endosc 1991; 5: 70 - 74

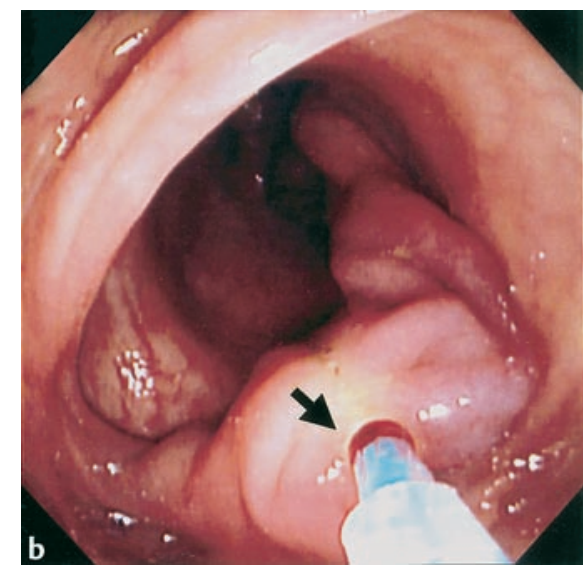

Corresponding Author

\section{Y. Tomiki, M.D.}

Dept. of Coloproctological Surgery, Juntendo University School of Medicine 2-1-1 Hongo, Bunkyo-ku Tokyo 113-8421

Japan

Fax: $\quad+81-3-3813-0731$

E-mail: tomiki@muh.biglobe.ne.jp
Figure 2 The detachable snare has been placed over a polyp that has a long stalk. $\mathbf{b}$ The red mark can be seen biting into the stalk of the polyp (arrow).

sensation and the color of the polyp (Figure 2). Using the monitor, the operator and assistant can confirm and agree on the tightness of the loop. The tightening maneuver is observed more easily than when the polypectomy is carried out without marking.

Using simple pen marking, this method allows safer polypectomy with detachable snares and may be regarded as a useful additional technique. 